\title{
96-DETC/MECH-1210
}

\section{ISOTROPIC COORDINATES, CIRCULARITY, AND BEZOUT NUMBERS: PLANAR KINEMATICS FROM A NEW PERSPECTIVE}

\author{
Charles W. Wampler \\ Manufacturing and Design Systems Dept. \\ General Motors R\&D Center, Bldg. 1-6 \\ 30500 Mound Road, Box 9055 \\ Warren, Michigan 48090 \\ USA
}

\begin{abstract}
It is commonly recognized that a convenient formulation for problems in planar kinematics is obtained by considering links to be vectors in the complex plane. However, scant attention has been paid to the natural interpretation of complex vectors as isotropic coordinates. These coordinates, often considered a special trick for analyzing four-bar motion, are in fact uniquely suited to two new techniques for analyzing polynomial systems: the BKK bound and the product-decomposition bound. From this synergistic viewpoint, a fundamental formulation of planar kinematics is developed and used to prove several new results, mostly concerning the degree and circularity of the motion of planar linkages. Useful for both analysis and synthesis of mechanisms, the approach both simplifies theoretical proofs and facilitates the numerical solution of mechanism problems.
\end{abstract}

\section{INTRODUCTION}

In formulating kinematic equations for a planar mechanism, one may use the fact that a point $(x, y)$ in Cartesian coordinates can be equivalently considered to be a point $x+i y$ in the complex plane, where $i^{2}=-1$. By the rules of complex arithmetic, it is seen that translation by $\left(d_{x}, d_{y}\right)$ and rotation by angle $\theta$ in the plane are given equivalently as

$$
\begin{aligned}
&\left(d_{x}+x \cos \theta-y \sin \theta, d_{y}+x \sin \theta+y \cos \theta\right) \\
& \Leftrightarrow\left(d_{x}+i d_{y}\right)+e^{i \theta}(x+i y) .
\end{aligned}
$$

Moreover, defining a complex number $z$ and its conjugate $\bar{z}$ as

$$
z=x+i y, \quad \bar{z}=x-i y
$$

one has that the squared length of $z$ is $z \bar{z}=x^{2}+y^{2}$. Because kinematics is primarily concerned with length-preserving (rigid-body) motions, a formulation in the complex plane is often convenient, and kinematicians frequently use this approach to model linkages. However, it is not uncommon to convert complex expressions into Cartesian coordinates, whereupon each complex vector equation yields two scalar equations (real and imaginary parts) and each length expression yields one scalar equation (the imaginary part of $z \bar{z}$ is identically zero). This article will show that, for reasons related to the phenomenon of circularity, a formulation in complex variables retains a simpler form and submits more readily to analysis than a Cartesian formulation.

Instead of considering $z$ and $\bar{z}$ to be a complex variable and its conjugate, it is just as valid to treat $(z, \bar{z})$ as a linear change of coordinates from $(x, y)$. The transformation is nonsingular, and in fact, its inverse has the simple form

$$
x=(z+\bar{z}) / 2, \quad y=(z-\bar{z}) /(2 i) .
$$

The term "isotropic coordinates" seems to have been first applied to the pair $(z, \bar{z})$ by Bricard (1927), but in fact the 
concept is older. Roberts (1875) briefly used isotropic coordinates in his proof of the triple generation of four-bar coupler curves. Darboux (1879) first formulated the fourbar closure conditions as two complex-conjugate equations, an approach which Morley (1921) adopted and developed further. Bricard's term and notation were used in further work on the four-bar by Haarbleicher (1933) and Groenman (1950). Isotropic coordinates have also been used to study six-bar and geared five-bar motion (Primrose and Freudenstein 1963, Primrose et al. 1967). Smith (1975) used a related formulation to evaluate velocities and accelerations of linkages.

When kinematic equations are studied in isotropic form, their structure is often more apparent than in Cartesian coordinates. This is especially true when applying several methods for counting the number of roots of a polynomial system, namely, the multi-homogeneous Bezout number, the BKK bound, and the product-decomposition bound. After presenting a general formulation for the kinematics of planar linkages in isotropic coordinates, this paper reviews root-counting techniques and examines circularity from this perspective. These developments are then used to prove several new results concerning the degree and circularity of the motion of planar linkages. The ideas are also profitably applied to problems of mechanism synthesis. In addition to theoretical proofs, the methods lead to practical numerical computations, particularly via polynomial continuation.

\section{ISOTROPIC FORMULATION}

In this section, a derivation of kinematic equations in isotropic coordinates for any planar linkage having rotational and/or prismatic joints is given. The methodology is then extended to planar linkages that include gear pairs.

\subsection{Basic formulation}

An unconstrained body moving in the plane has 3 degrees of freedom: two translations and one rotation. Accordingly, for each of the $n-1$ moving links of an $n$-bar linkage, there are three motion variables: for $k=1, \ldots, n-1$, let two variables, $z_{k}$ and $\bar{z}_{k}$, be isotropic coordinates for the translation, and let $\Theta_{k}$ be the angle of rotation. Rotation in the complex plane involves the quantity $e^{i \Theta_{k}}$, which is transcendental, so to keep the formulation algebraic, introduce a pair of new variables, $\theta_{k}, \bar{\theta}_{k}$, defined as

$$
\theta_{k}=e^{i \Theta_{k}}, \quad \bar{\theta}_{k}=e^{-i \Theta_{k}}, \quad k=1, \ldots, n-1,
$$

with the additional equation

$$
\theta_{k} \bar{\theta}_{k}=1, \quad k=1, \ldots, n-1 .
$$

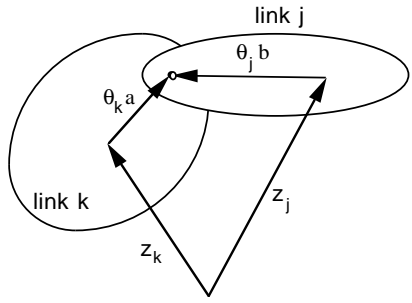

(a)

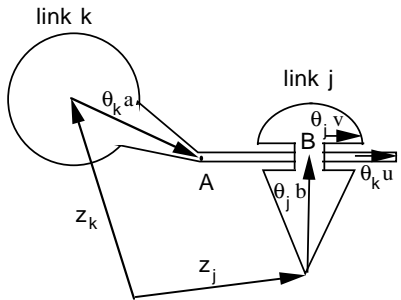

(b)
Figure 1. Isotropic formulation for rotational (a) and prismatic (b) joints.

Thus, for each moving link $k$, there are four variables $z_{k}, \bar{z}_{k}, \theta_{k}, \bar{\theta}_{k}$ and one polynomial relation, Eq.(4). For the fixed (ground) link, one may assume without loss of generality that $z_{0}=\bar{z}_{0}=0$ and $\theta_{0}=\bar{\theta}_{0}=1$.

2.1.1 Rotational joints. A planar rotational joint centered on point $A$ of one link and on point $B$ of another link is modeled by setting the coordinates of $A$ equal to the coordinates of $B$. Suppose that when link $k$ is in its reference position, point $A$ of the link has isotropic coordinates $(a, \bar{a})$. By Eq.(1), when the link is displaced, the new isotropic coordinates of $A$ are $\left(z_{k}+\theta_{k} a, \bar{z}_{k}+\bar{\theta}_{k} \bar{a}\right)$, and similarly for $B$. Accordingly, as illustrated in Fig. 1(a), the pin joint between $A$ and $B$ gives the pair of equations:

$$
z_{k}+\theta_{k} a=z_{j}+\theta_{j} b, \quad \bar{z}_{k}+\bar{\theta}_{k} \bar{a}=\bar{z}_{j}+\bar{\theta}_{j} \bar{b} .
$$

There are two such equations for each rotational joint in the linkage.

2.1.2 Prismatic joints. To model a prismatic joint, one must equate a line in link $k$ with a line in link $j$, (see Fig. 1(b)). For this purpose, let $(u, \bar{u})$ be the isotropic coordinates for a unit vector in link $k$, when the link is in its reference position. Then, after displacement of the link, the new isotropic coordinates of the unit vector are $\left(\theta_{k} u, \bar{\theta}_{k} \bar{u}\right)$. If unit vectors $(u, \bar{u})$ of link $k$ and $(v, \bar{v})$ of link $j$ are each parallel to the line of action of a prismatic joint between those links, then

$$
\theta_{k} u=\theta_{j} v, \quad \bar{\theta}_{k} \bar{u}=\bar{\theta}_{j} \bar{v}
$$

Since $u$ and $v$ are unit vectors, $u \bar{u}=1$ and $v \bar{v}=1$, and so $\theta_{k} \bar{\theta}_{k}=(v / u) \theta_{j}(\bar{v} / \bar{u}) \bar{\theta}_{j}=\theta_{j} \bar{\theta}_{j}$. Hence, one of Eqs.(4) is superfluous and can be dropped. Eq.(6) ensures that the line in link $k$ is parallel to the line in link $j$, but they are 
also coincident. Accordingly, if point $A$ of link $k$ and point $B$ of link $j$ lie on the respective lines defining the joint, then the line segment $\overline{A B}$ must be a scalar multiple, $s_{k} \in \mathbb{R}$, of the unit vector along the joint:

$$
\begin{aligned}
& \left(z_{k}+\theta_{k} a\right)-\left(z_{j}+\theta_{j} b\right)=s_{k} \theta_{k} u, \\
& \left(\bar{z}_{k}+\bar{\theta}_{k} \bar{a}\right)-\left(\bar{z}_{j}+\bar{\theta}_{j} \bar{b}\right)=s_{k} \bar{\theta}_{k} \bar{u},
\end{aligned}
$$

where the fact that the conjugate of a scalar is the scalar itself, $\bar{s}_{k}=s_{k}$, has been used. The distance $s_{k}$ is the joint variable for the prismatic joint. If the joint is the input for the mechanism, $s_{k}$ is known, otherwise it may be eliminated to get

$\bar{\theta}_{k} \bar{u}\left[\left(z_{k}+\theta_{k} a\right)-\left(z_{j}+\theta_{j} b\right)\right]-\theta_{k} u\left[\left(\bar{z}_{k}+\bar{\theta}_{k} \bar{a}\right)-\left(\bar{z}_{j}+\bar{\theta}_{j} \bar{b}\right)\right]=0$.

With the addition of the three equations $(6,7)$ and the elimination of one of Eqs.(4), each prismatic joint contributes a net increase of two equations.

2.1.3 Gear pairs. Finally, consider the case of a gear pair. Suppose links $m$ and $n$ are a gear pair pinned to link $k$, having gear ratio $p / q$, where $p$ and $q$ are relatively prime. Accordingly, in the frame of link $k$, link $m$ rotates $q$ times while link $n$ rotates $p$ times. (For a negative gear ratio, one of $p$ or $q$ is taken as negative.) In addition, links $m$ and $n$ may have initial rotations of $\Phi$ and $\Psi$ before the gear mesh is engaged. The total rotation of each gear is the sum of the initial offset, the rotation of the carrier link, and the internal rotation of the gear set. By introducing a new angle $\Gamma$ associated with the gear mesh, one may express the rotation of link $m$ as $\Theta_{m}=\Phi+\Theta_{k}+q \Gamma$ and the rotation of link $n$ as $\Theta_{n}=\Psi+\Theta_{k}+p \Gamma$. Consequently, with the notations $\theta_{m}=e^{i \Theta_{m}}, \phi=e^{i \Phi}, \theta_{n}=e^{i \Theta_{n}}, \psi=e^{i \Psi}, \gamma=e^{i \Gamma}$, and their conjugates, the rotations are given in isotropic coordinates by

$$
\begin{aligned}
\theta_{m} & =\phi \theta_{k} \gamma^{q}, & \bar{\theta}_{m} & =\bar{\phi} \bar{\theta}_{k} \bar{\gamma}^{q}, \\
\theta_{n} & =\psi \theta_{k} \gamma^{p}, & \bar{\theta}_{n} & =\bar{\psi} \bar{\theta}_{k} \bar{\gamma}^{p},
\end{aligned}
$$

where $\gamma$ and $\bar{\gamma}$ are new variables which obey the relation

$$
\gamma \bar{\gamma}=1
$$

From these, Eqs.(4) are automatically satisfied for links $m$ and $n$, hence the gear pair has introduced two new variables $(\gamma, \bar{\gamma})$ and contributed a net increase of 3 equations. Accordingly, the gear mesh removes one degree of freedom, as it should.
2.1.4 Mobility. Through the use of Eqs.(4-9), the kinematics of any planar linkage with rotational, prismatic, and gear pair joints can be modeled in terms of isotropic polynomial equations. For an $n$-bar linkage with $r$ rotational joints, $p$ prismatic joints and $g$ gear meshes, there are $(n-1)+2 r+2 p+3 g$ equations in $4(n-1)+2 g$ variables, which gives the familiar mobility relation

$$
M=3(n-1)-2 r-2 p-g .
$$

2.1.5 Reduction. For a given linkage, it is easy to reduce the system of equations, mainly due to the simple, linear form of Eqs. $(5,6)$. Furthermore, as is shown below in $\S 5.3$, for an RR binary ${ }^{1}$ link, one can often avoid introducing the associated link variables.

2.1.6 Example. As an illustration of this isotropic formulation, consider the inverted geared five-bar shown in Fig. 2. Links 1 and 3 are a gear pair, pinned to link 2 at points $A$ and $B$, resp. An extension of link 1 is pinned to ground (link 5) at point $O$, and a line of link 3 rides in a slot on link 4, which is in turn pinned to ground at point $E$. The origins of links 1 to 4 are taken as points $O$, $A, B$, and $E$, resp., and the origin for the ground link is also taken as $O$. Vectors $a, b, c, d, e$ give the location of points $A, B, C, D, E$ in the reference frame of links $1,2,3,4,5$, resp. Vectors $v$ and $u$ are unit vectors along the two members of the prismatic joint, when links 3 and 4 are in their reference position. The ratio of the number of teeth on gear 1 to the number on gear 3 is $p: q$, reduced to lowest terms. At initial assembly, links 1 and 2 are in reference orientation but link 3 is given a rotation of $\Psi$ before engaging the gears.

From the foregoing description of the parts, the equations describing the kinematics of the assembled mechanism are easily written out using the isotropic formulation as follows. Per Eq. (4), there are the unit vector equations:

$$
\theta_{k} \bar{\theta}_{k}=1, \quad k=1,2,3,4 \text {. }
$$

By Eq. (5), rotational joints at $O, A, B, E$ give:

$$
\begin{array}{ll}
z_{1}=0, & \bar{z}_{1}=0, \\
z_{2}=\theta_{1} a, & \bar{z}_{2}=\bar{\theta}_{1} \bar{a}, \\
z_{3}=z_{2}+\theta_{2} b, & \bar{z}_{3}=\bar{z}_{2}+\bar{\theta}_{2} \bar{b}, \\
z_{4}=e, & \bar{z}_{4}=\bar{e} .
\end{array}
$$

\footnotetext{
${ }^{1}$ A binary link connects to two adjacent links, a tertiary link connects to three adjacent links, and so on for quaternary links and higher.
} 


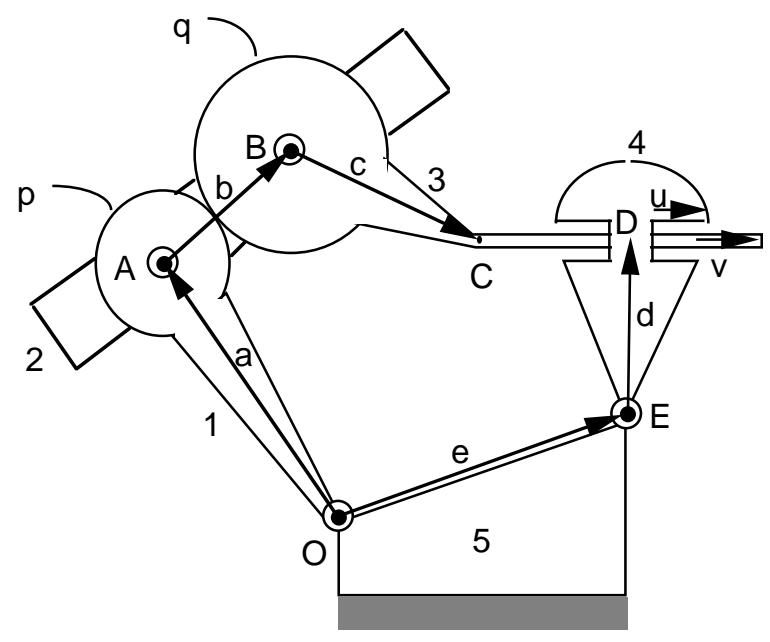

Figure 2. A geared five-bar mechanism.

The prismatic joint gives, by Eqs. $(6,7)$,

$$
\begin{gathered}
\theta_{3} v=\theta_{4} u, \quad \bar{\theta}_{3} \bar{v}=\bar{\theta}_{4} \bar{u}, \\
\bar{\theta}_{4} \bar{u}\left[\left(z_{4}+\theta_{4} d\right)-\left(z_{3}+\theta_{3} c\right)\right] \\
-\theta_{4} u\left[\left(\bar{z}_{4}+\theta_{4} \bar{d}\right)-\left(\bar{z}_{3}+\bar{\theta}_{3} \bar{c}\right)\right]=0 .
\end{gathered}
$$

Finally, the gear pair introduces the following:

$$
\begin{array}{lrl}
\theta_{1}=\theta_{2} \gamma^{q}, & \bar{\theta}_{1}=\bar{\theta}_{2} \bar{\gamma}^{q}, \\
\theta_{3}=\psi \theta_{2} \gamma^{-p}, \quad \bar{\theta}_{3}=\bar{\psi} \bar{\theta}_{2} \bar{\gamma}^{-p}, & \gamma \bar{\gamma}=1,
\end{array}
$$

where the negative exponents reflect the fact that the gears rotate in opposition. Of the unit vector equations, only $\theta_{2} \bar{\theta}_{2}=1$ is independent, because the others are implied by the joint equations and the assumption that $v \bar{v}=u \bar{u}=1$. Due to the simple form of the basic equations, it is straightforward to make substitutions into the prismatic joint equation and obtain a single equation in two variables $\theta_{2}, \gamma$ :

$$
\begin{array}{r}
\bar{\psi} \bar{v} \theta_{2}^{-1} \gamma^{p}\left[\left(e+(v / u) \psi \theta_{2} \gamma^{-p} d\right)-\left(\theta_{2} \gamma^{q} a+\theta_{2} b+\psi \theta_{2} \gamma^{-p} c\right)\right] \\
-\psi v \theta_{2} \gamma^{-p}\left[\left(\bar{e}+(\bar{v} / \bar{u}) \bar{\psi} \theta_{2}^{-1} \gamma^{p} \bar{d}\right)\right. \\
\left.-\left(\theta_{2}^{-1} \gamma^{-q} \bar{a}+\theta_{2}^{-1} \bar{b}+\bar{\psi} \theta_{2}^{-1} \gamma^{p} \bar{c}\right)\right]=0
\end{array}
$$

Given a value of $\gamma$, this equation becomes a quadratic in $\theta_{2}$. After solving for $\theta_{2}$, the positions and orientations of all the links are easily found. With one sweep of $\gamma$ around the unit circle, the entire motion of the linkage can be computed. On the other hand, if $\theta_{2}$ is taken as given, the equation is a polynomial of degree $2(p+q)$ in $\gamma$.

\section{BEZOUT NUMBERS}

While isotropic coordinates are convenient for derivations of the sort demonstrated in $\S 2.1 .6$, the biggest advantage in using this formulation is its compatibility with modern methods of counting the number of solutions of polynomial equations. This section briefly reviews the theory used in this paper.

Kinematicians are familiar with the idea that "general" examples from a family of polynomial systems have a constant number of solutions in complex numbers. Hence, one may say that the inverse kinematic problem of a general six-revolute serial-link robot has 16 solutions. At the same time, it is well-known that such problems have special cases where the number of solutions may change; for example, if three successive joint axes of the robot arm intersect in a point, then the inverse kinematic problem has only 8 solutions.

These ideas are made precise by the definition of a Bezout number. Suppose we have a system of $n$ functions $P(q, x)$ that are polynomial in $n$ complex variables $x \in \mathbb{C}^{n}$ and analytic in $m$ complex parameters $q \in \mathbb{C}^{m}$. Then, for almost all $q \in \mathbb{C}^{m}$, the system of polynomial equations $P(q, x)=0$ has a constant number, $N$, of nonsingular solutions, although it may have a smaller number for parameters satisfying extra analytic equations. The existence of 16 solutions for the general $6 \mathrm{R}$ robot problem and the reduction to 8 solutions when the problem is restricted to intersecting wrist axes are examples of this phenomenon. Let $N$ be called the Bezout number of $P(q, x)$, sometimes written $N(P)$. In addition to restricting the parameter space, one may also ban solutions that satisfy some additional algebraic equations, in which case the Bezout number may decrease. In any case, the Bezout number for a system is an upper bound on the number of nonsingular solutions of any special case of that system. (For more details, see Morgan and Sommese 1989.)

A well-known example of a Bezout number is provided by Bezout's Theorem, stating that a system of $n$ polynomial equations of degree $d_{1}, \ldots, d_{n}$ in $n$ variables has at most $\prod_{j=1}^{n} d_{j}$ nonsingular solutions on $\mathbb{C}^{n}$. Here, the parameters $q$ are just the coefficients of the general polynomials of degree $d_{1}, \ldots, d_{n}$.

\subsection{Product decomposition}

A technique called product decomposition (Morgan, et al 1995) can be used to determine Bezout numbers for systems described using the following notation. For polynomials $u_{1}, \ldots, u_{j}$, let $\left\langle u_{1}, \ldots, u_{j}\right\rangle$ represent the vector space of all linear combinations of $u_{1}, \ldots, u_{j}$. Also, define the 
product of two such spaces as follows:

$$
\begin{aligned}
& \left\langle u_{1}, \ldots, u_{j}\right\rangle\left\langle v_{1}, \ldots, v_{k}\right\rangle= \\
& \left\langle u_{1} v_{1}, \ldots, u_{1} v_{k}, \quad \ldots \quad, u_{j} v_{1}, \ldots, u_{j} v_{k}\right\rangle,
\end{aligned}
$$

where the right-hand side is meant to include all possible pairs of elements from the two groups on the left.

The product-decomposition bound does not directly give a Bezout number, but rather gives an upper bound on one Bezout number in terms of another. Suppose that in the system $f(x)=\left(f_{1}(x), \ldots, f_{n}(x)\right)=0$, the first polynomial has the structure $f_{1} \in\left\langle u_{1}, \ldots, u_{j}\right\rangle\left\langle v_{1}, \ldots, v_{k}\right\rangle$. Then, according to Morgan, et al. (1995),

$$
\begin{aligned}
& N(f) \leq N\left(\left[\left(q_{1} u_{1}+\cdots+q_{j} u_{j}\right)\left(q_{j+1} v_{1}+\cdots+q_{j+k} v_{j}\right)\right.\right. \\
&\left.\left.f_{2}, \ldots, f_{n}\right]\right) \\
& \leq N\left(\left[q_{1} u_{1}+\cdots+q_{j} u_{j}, f_{2}, \ldots, f_{n}\right]\right) \\
&+N\left(\left[q_{j+1} v_{1}+\cdots+q_{j+k} v_{k}, f_{2}, \ldots, f_{n}\right]\right)
\end{aligned}
$$

where $q_{1}, \ldots, q_{j+k}$ are generic coefficients. The same result can be applied several times to break $f_{1}$ into more than two products, and by permuting the order of the polynomials, $f_{2}, \ldots, f_{n}$ can be decomposed as well. The usefulness of the product decomposition is that one can get an upper bound on $N(f)$ by finding the Bezout number of the simpler subsystems displayed in (12). Moreover, the system on the right-hand side of (11) can be used as a start system in the solution of $f=0$ by polynomial continuation.

\subsection{Multihomogeneous Bezout number}

The multi-homogeneous Bezout number is a consequence of results described in (Shafarevich 1977), and was stated formally in (Morgan and Sommese 1987), where it was also first shown relevant to kinematics. It can also be derived using the product-decomposition formula just described. Suppose that a system of polynomials $f=$ $\left(f_{1}, \ldots, f_{n}\right)$ has the structure

$$
f_{i} \in\left\langle 1, x_{11}, \ldots, x_{1 k_{1}}\right\rangle^{d_{i 1}} \ldots\left\langle 1, x_{m 1}, \ldots, x_{m k_{m}}\right\rangle^{d_{i m}}
$$

where the partitioning of the variables is the same across all $f_{i}$, but the degrees may vary. By the product decomposition theorem, a bound on the Bezout number of system $f$ can be found as the sum of the Bezout numbers for a collection of linear systems. Most of these will be overdetermined and have no solution, leaving $N(f)$ to be the number of wellposed linear systems. This can be seen to be the coefficient of $\alpha_{1}^{k_{1}} \cdots \alpha_{m}^{k_{m}}$ in the combinatorial product $\prod_{i=1}^{n}\left(d_{i 1} \alpha_{1}+\cdots+\right.$ $\left.d_{i m} \alpha_{m}\right)$. The 1-homogeneous Bezout number is the same as that given by Bezout's Theorem.

\subsection{BKK Bezout number}

Another useful Bezout number is the BKK bound (Bernshtein 1975), in which each polynomial is summarized by a list of its monomials, for example, $f_{1}=\left\langle m_{1}, \ldots, m_{k}\right\rangle$, for monomials $m_{1}, m_{2}, \ldots, m_{k}$. The exponents appearing in these monomials determine polytopes in $n$-dimensional space. With the parameters $q$ as the coefficients of the mononomials and the solution space as $x \in(\mathbb{C}-\{0\})^{n}$ (that is, any solution for which one or more of the variables is zero does not count), the BKK bound gives the Bezout number as a geometric function of the polytopes, called the mixed volume. The mixed volume can be calculated by hand only for the simplest cases, but computer algorithms are available (Verschelde et al. 1994, Huber and Sturmfels 1995). These references also give homotopies for solving polynomial systems numerically by continuation, with the number of solution paths equal to the BKK bound.

\section{CIRCULARITY}

Because Bezout's Theorem is merely an upper bound, the number of intersections between two planar curves is often less than the product of their degrees. This is especially true of the curves studied in kinematics, which often possess a property called circularity. We first look at the phenomenon in the simplest case, the intersection of two circles, and then proceed to the general case. The issue of accounting for circularity is greatly facilitated by the use of isotropic coordinates.

\subsection{Intersection of two circles}

By Bezout's theorem, two quadratic curves in the plane have at most $2 \cdot 2=4$ points of intersection, yet it is an elementary fact that two circles, regardless of their size or position, can have at most two nonsingular points in common. The classical approach to account for this discrepancy is as follows. The equation for a circle centered at $c=c_{x}+i c_{y}$ of radius $r$ is

$$
(z-c)(\bar{z}-\bar{c})=r^{2} .
$$

To homogenize the equation, let $z=Z / W$ and $\bar{z}=\bar{Z} / W$, and clear denominators to get

$$
(Z-c W)(\bar{Z}-\bar{c} W)=r^{2} W^{2}
$$

One sees that every circle contains two points at infinity: $(Z, \bar{Z}, W)=(1,0,0)$ and $(0,1,0)$. Since these points are common to all circles, they are called "isotropic points". (As a point of reference, note that in homogenized Cartesian 
coordinates, the isotropic points are given by $(X, Y, W)=$ $(1, \pm i, 0)$, which are the two roots of $X^{2}+Y^{2}=0$. $)$ Because of these two roots at infinity, the intersection of two circles contains at most $4-2=2$ finite roots.

Isotropic coordinates allow us to use alternatives to Bezout's theorem that exclude the isotropic points when counting intersections. Since each circle has the product structure $\langle z, 1\rangle\langle\bar{z}, 1\rangle$, the 2-homogeneous Bezout number for the intersection of two circles is the coefficient of $\alpha_{1} \alpha_{2}$ in $\left(\alpha_{1}+\alpha_{2}\right)^{2}$, which is 2 . In a 2-homogenization of the circle equations,

$$
(Z-c W)(\bar{Z}-\bar{c} \bar{W})=r^{2} W \bar{W}
$$

the isotropic solutions no longer exist, because the indeterminates $(Z, W)=(0,0)$ and $(\bar{Z}, \bar{W})=(0,0)$ are not allowed. Notice that to get a sharp 2-homogeneous Bezout number, it is essential to use isotropic coordinates: in Cartesian coordinates, the 2-homogeneous Bezout number is 8 .

\subsection{Curves with circularity}

If the isotropic points only arose in connection with circle intersections, they would not warrant much attention, but it happens that most curves generated by planar linkages pass through the isotropic points one or more times. Thus, in counting the number of intersections of such curves, the isotropic points figure prominently. In a 1homogeneous treatment, the multiplicity of the intersection of a curve with the isotropic points is called the circularity of the curve. The standard formula for the circularity of a real ${ }^{2}$ polynomial $f(x, y)$ in Cartesian coordinates as follows. For $k=0, \ldots, d$, where $d$ is the degree of $f$, let $c_{k}$ be the largest integer such that the terms of degree $k$ in $f$ are members of $\left\langle x^{2}+y^{2}\right\rangle^{c_{k}}\langle x, y\rangle^{k-2 c_{k}}$. Then, the circularity $c$ of $f$ is

$$
c=\min _{0 \leq k \leq d}\left(d-k+c_{k}\right) .
$$

The sense behind this definition is clearer in isotropic coordinates. Let $g(z, \bar{z})=f((z+\bar{z}) / 2,(z-\bar{z}) /(2 i))$ be the same curve represented in isotropic coordinates. Since the coefficients in $f$ are real, the complex conjugate of $f$ for real $(x, y)$ is equal to $f$, and hence the same is true for $g$. This implies that any term $\alpha z^{p} \bar{z}^{q}$ must be matched by a conjugate term $\bar{\alpha} z^{q} \bar{z}^{p}$ so that the sum $\alpha z^{p} \bar{z}^{q}+\bar{\alpha} z^{q} \bar{z}^{p}$ is invariant under conjugation. Accordingly, the degree of $g$

\footnotetext{
${ }^{2}$ For complex coefficients, the two isotropic points may have unequal multiplicities, but this is not of interest for mechanism work.
}

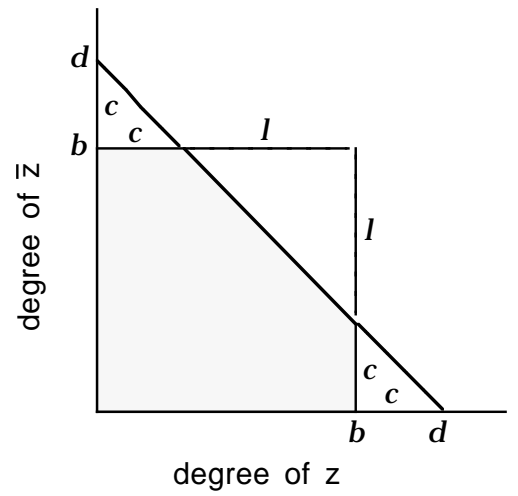

Figure 3. Monomials for a plane curve of degree $d$ and bidegree $b$ lie at the integer lattice points in the shaded region. Circularity $c$ and linearity $\ell$ are indicated.

with respect to $z$ is equal to the degree of $g$ with respect to $\bar{z}$, and we will call this number the isotropic bidegree $b$ of $g$. The $k$ th-degree term of $g$ is of the form $z^{c_{k}} \bar{z}^{c_{k}}\langle z, \bar{z}\rangle^{k-2 c_{k}}$, so the bidegree is

$$
b=\max _{0 \leq k \leq d}\left(k-c_{k}\right)=d-c .
$$

Alternatively, one may simply note that $g$ has the productdecomposition form:

$$
g \in\langle z, 1\rangle^{c}\langle\bar{z}, 1\rangle^{c}\langle z, \bar{z}, 1\rangle^{\ell}
$$

where $c$ is the circularity and $\ell$ is a new quantity that we will call the linearity. The degree and bidegree of $g$ are $d=2 c+\ell$ and $b=c+\ell$, respectively.

The relationships between circularity, linearity, degree and bidegree can be visualized by plotting the polytope associated to the monomials in $g$, as shown in Fig. 3. In isotropic coordinates, the circularity of a curve is determined by the shape of its polytope, whereas in Cartesian coordinates, it is determined by relationships between coefficients (e.g., $x^{2}+y^{2}$ has equal coefficients on the monomials $x^{2}$ and $\left.y^{2}\right)$. This is why isotropic coordinates and the BKK bound work so well together.

4.2.1 1-Homogeneous viewpoint. Generalizing the argument used by Bricard (1927) in analyzing the four-bar coupler curve, the multiplicity of the isotropic points can be found as follows. The intersection of $g$ with a linear equation in $\bar{z}$, i.e., with $f=0$ where $f \in\langle\bar{z}, 1\rangle$, is a curve of degree $c+\ell$ in $z$, and so there are only $c+\ell$ finite roots instead of $d=2 c+\ell$. Hence, in a 1-homogeneous formulation, 
the point at infinity along the $z$-axis must be a root of multiplicity $c$, and similarly for the other isotropic point along the $\bar{z}$-axis. Thus, in the intersection between two curves of degree $d_{1}$ and $d_{2}$ and circularity $c_{1}$ and $c_{2}$, respectively, the isotropic points each appear with multiplicity $c_{1} c_{2}$, so the number of nonsingular finite intersections is at most

$$
N=d_{1} d_{2}-2 c_{1} c_{2}
$$

\subsubsection{2-Homogeneous viewpoint. From}

a 2 -homogeneous viewpoint, the result is the same, but the interpretation is different. The 2-homogeneous Bezout number for the intersection between a curve with bidegrees $\left(b_{1}, b_{1}\right)$ and a curve with bidegrees $\left(b_{2}, b_{2}\right)$ is the coefficient of $\alpha_{1} \alpha_{2}$ in $\left(\alpha_{1}^{b_{1}}+\alpha_{2}^{b_{1}}\right)\left(\alpha_{1}^{b_{2}}+\alpha_{2}^{b_{2}}\right)$, which is $2 b_{1} b_{2}$. But upon 2-homogenizing the equations, one finds that there is a root at infinity $\{(Z, W),(\bar{Z}, \bar{W})\}=\{(1,0),(1,0)\}$ of multiplicity $\ell_{1} \ell_{2}$, Hence, the number of finite intersections is

$$
N=2 b_{1} b_{2}-\ell_{1} \ell_{2}
$$

4.2.3 Product decomposition. Considering the problem once more using the product-decomposition theorem, one has the polynomials $g_{1} \in\langle z, 1\rangle^{c_{1}}\langle\bar{z}, 1\rangle^{c_{1}}\langle z, \bar{z}, 1\rangle^{\ell_{1}}$ and $g_{2} \in\langle z, 1\rangle^{c_{2}}\langle\bar{z}, 1\rangle^{c_{2}}\langle z, \bar{z}, 1\rangle^{\ell_{2}}$. For the purpose of counting roots, the intersection of $g_{1}=0$ with $g_{2}=0$ can be broken into 9 subsystems according to the product structure. Among these, the subsystem $\left\{\langle z, 1\rangle^{c_{1}}=0,\langle z, 1\rangle^{c_{2}}=0\right\}$ has no roots and neither does $\left\{\langle\bar{z}, 1\rangle^{c_{1}}=0,\langle\bar{z}, 1\rangle^{c_{2}}=0\right\}$, but the remaining subsystems all contribute roots totaling to

$$
N=2\left(c_{1} c_{2}+c_{1} \ell_{2}+c_{2} \ell_{1}\right)+\ell_{1} \ell_{2} \text {. }
$$

The BKK bound will also yield this result.

4.2.4 Equivalence. Although Eqs.(14,15,16) do not look the same, one can use the relations $d_{k}=2 c_{k}+\ell_{k}$ and $b_{k}=c_{k}+\ell_{k},(k=1,2)$, to show that they are all in exact agreement. The combination of isotropic coordinates with the product-decomposition theorem or the BKK bound give a sharp Bezout number without a separate accounting for isotropic roots at infinity.

\section{KINEMATIC ANALYSIS OF LINKAGES}

The scope of the remainder of this paper will be narrowed according to the following definitions.

Definition 1. A planar linkage is a mechanism with only rotational and prismatic joints.
Definition 2. An exactly-constrained linkage is one whose number of degrees of freedom of motion is equal to its mobility as calculated from Eq. (10).

In particular, this definition excludes over-constrained linkages, whose degrees of freedom of motion exceed their mobility. By Eq. (10), a mobility-1 exactly-constrained linkage $(M=1, g=0)$ has $r+p=(3 n-4) / 2$ rotational and prismatic joints, and since the number of joints is an integer, $n$ must be even.

By "kinematic analysis" of a linkage, we mean the problem of characterizing motion in terms of either an input/output relationship or a curve traced by the linkage:

- Input/output analysis of a linkage answers the following question: for a given value of $\Theta_{j}$, what values can $\Theta_{k}$ take? The number of such values, herein referred to as the input/output degree, depends on the topology of the linkage and generally varies depending on which links $j$ and $k$ are designated as input and output. The input link is herein assumed to be connected to the ground link.

- Tracing curve ${ }^{3}$ analysis seeks to delineate the geometric properties of the locus traced by a selected point on one of the links. Chief among these properties are the degree and circularity of the curve, which are important in determining the number of intersections the curve may have with other plane curves.

In the section to follow, these two problems are seen to be interrelated and in fact, correspond to questions concerning the assembly modes of certain immobile structures. From this unified perspective, we proceed in the succeeding sections to apply the isotropic formulation to answer questions concerning degree and circularity.

\subsection{Structures, robots and linkages}

Before proceeding with the analysis of 1-degree-offreedom linkages, it will be useful to note certain correspondences between questions regarding the motion of linkages and questions regarding the assembly of structures. These questions are also closely related to the forward kinematic problem for planar in-parallel robots. For this discussion, the following definition is used.

Definition 3. A structure ${ }^{4}$ is an exactly-constrained linkage having mobility $M=0$.

Accordingly, all structures must have an odd number of links. The main question in analyzing a structure is to find

\footnotetext{
${ }^{3}$ Tracing curves are sometimes called coupler curves by other kinematicians (e.g., Primrose et al. 1967).

${ }^{4}$ A more precise term would be be "statically determinate structure."
} 


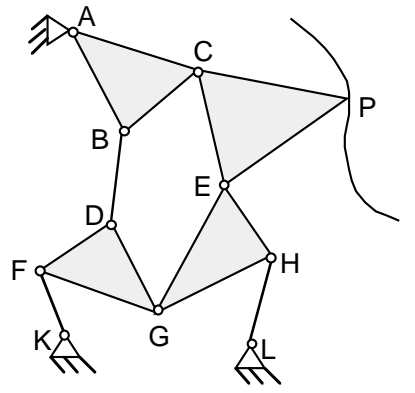

(a)

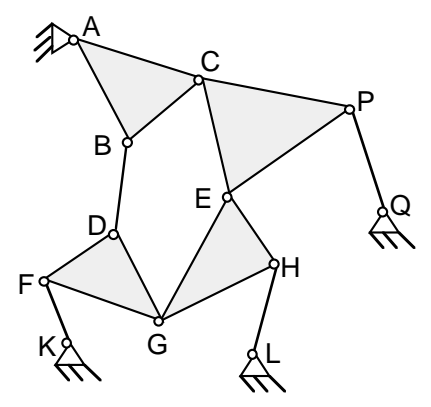

(c)

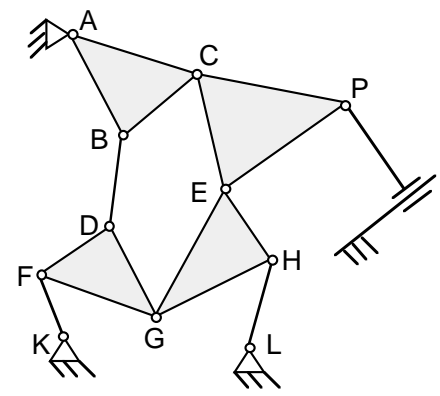

(b)

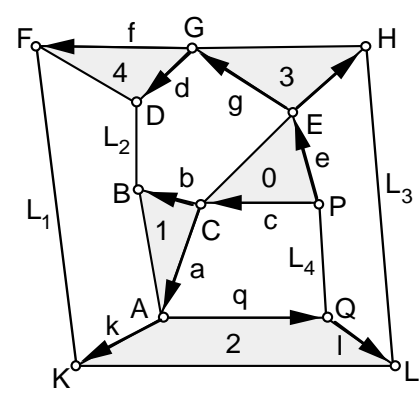

(d)

Figure 4. An eight-bar linkage and related nine-bar structures.

all assembly configurations, given the link dimensions and their interconnections. For example, three binary links of prescribed length can be assembled into a planar triangle in two ways, one a mirror image of the other.

Structures are closely related to the main problems in linkage analysis. In the case of input/output relations, one sees that for each value of the input angle, the input link and ground link can be temporarily viewed as a single link. The number of assembly modes of the resulting $(2 k-1)$ bar structure is seen to be the same as the degree of the input/output relation for the original $2 k$-bar linkage.

The analysis of tracing curves is related to structures as illustrated in Fig. 4. An eight-bar linkage is shown in Fig. 4(a), along with a portion of the tracing curve associated to point $P$. The degree of the tracing curve is equal to the number of times it intersects an arbitrary line (Eq.14). This corresponds to the number of assemblies of the structure obtained by adding an $R P$ binary link between $P$ and ground, as shown in Fig. 4(b). Similarly, the bidegree of the tracing curve is equal to one-half the number of times it intersects an arbitrary circle (Eq.15), which corresponds to adding an $R R$ binary link between $P$ and ground, such as link $P Q$ in Fig. 4(c). Fig. 4(d) is a re-arrangement of Fig. 4(c), emphasizing the quaternary nature of the ground link $A K L Q$. The straightforward generalization of these constructions shows that the analysis of tracing curves for

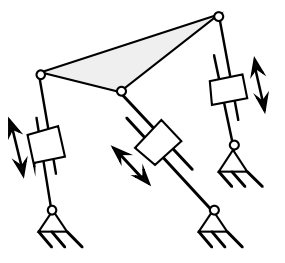

(a)

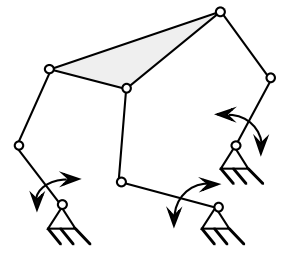

(b)

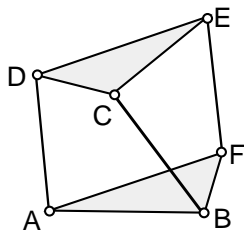

(c)
Figure 5. Two in-parallel planar manipulators $(a, b)$ whose forward kinematics reduce to the pentad structure (c). Arrows indicate actuated joints.

a $2 k$-bar linkage is related to the modes of assembly of certain $(2 k+1)$-bar structures. The specific example of Fig. 4 will be examined further in $\$ 5.3$.

Putting together the observations of the last two paragraphs, one sees that the analysis of a tracing curve for a $2 k$-bar linkage determines the number of assemblies of certain $(2 k+1)$-bar structures, which in turn determines the input/output degree of several $(2 k+2)$-bar linkages.

Structures are also related to robot kinematics. A planar robot is, typically, a mobility- 3 exactly-constrained linkage, with three actuated joints. When the actuated joints are held constant, the device becomes a structure, with the end-effector held in a fixed location. (See Fig. 5.) Accordingly, the forward kinematic problem of determining the location of the end-effector given the input joint values is a question of finding the assembly modes of the associated structure.

\subsection{General linkages}

In this section, we establish bounds on the basic problems of kinematic analysis considering only the number of links in the mechanism. The main result depends on the following lemma.

Lemma 1. The D-homogeneous Bezout number for $k+l$ polynomials that are bi-linear in $x_{1} \in \mathbb{C}^{k}$ and $x_{2} \in \mathbb{C}^{l}$ is $\left(\begin{array}{c}k+l \\ k\end{array}\right)$.

Proof. By the multi-homogeneous Bezout formula given in $\S 3$, the Bezout number is the coefficient of $\alpha_{1}^{k} \alpha_{2}^{l}$ in $\left(\alpha_{1}+\alpha_{2}\right)^{k+l}$. From this, the lemma is a consequence of the binomial theorem.

The following theorem places bounds on the degree and bidegree of a curve traced by a planar linkage. As illustrated in Fig. 3, the degree and bidegree define a polygon inside of which the monomials of the polynomial equation describing the curve must lie. Remember that the degree $d$ and bidegree $b$ determine the circularity as $c=d-b$ (Eq.13). 
Theorem 1. A $(2 k+1)$-bar structure can be assembled at most $\left(\begin{array}{c}2 k \\ k\end{array}\right)$ ways.

Corollary 1. The curve traced by any point of a mobility-1 exactly-constrained $2 k$-bar planar linkage has degree $d \leq$ $\left(\begin{array}{c}2 k \\ k\end{array}\right)$ and bidegree $b \leq \frac{1}{2}\left(\begin{array}{c}2 k \\ k\end{array}\right)$.

Corollary 2. The input/output relation of a mobility-1 exactly-constrained $2 k$-bar linkage has degree $d \leq\left(\begin{array}{c}2 k-2 \\ k-1\end{array}\right)$.

Proof. For the isotropic formulation of $\S 2$, it is natural to adopt a 2-homogeneous formulation wherein the primary variables $\left\{z_{l}, \theta_{l}\right\}(l=1, \ldots, 2 k)$ are placed in one subset, and the conjugate variables $\left\{\bar{z}_{l}, \bar{\theta}_{l}\right\}(l=1, \ldots, 2 k)$ are placed in the other. There are $2 k$ unit vector equations of the form of Eq.(4) (one link is fixed). In addition, in the case that all joints are rotational, there are $6 k$ linear equations of the form Eq.(5). Of these, $3 k$ involve only the primary variables and $3 k$ involve the conjugate variables. The situation does not change when prismatic joints are present, as each prismatic joint also introduces two linear equations (6) and replaces one of the unit vector equations with a bilinear equation of the form ( 7 ). The linear equations can be used to reduce the system to $2 k$ equations, each bilinear with respect to $k$ primary and $k$ conjugate variables. By Lemma 1 , the system of equations has at most $\left(\begin{array}{c}2 k \\ k\end{array}\right)$ solutions, hence Theorem 1 is proven. As discussed in $\$ 5.1$, the degree and bidegree of a tracing curve can be determined by considering the $(2 k+1)$-bar structures obtained by adding either an $R P$ binary link or $R R$ binary link connecting the tracing point to ground. By Eqs. $(14,15)$, this number is equal to the degree and equal to twice the bidegree of the tracing curve, hence Corollary 1 follows. Corollary 2 is a consequence of the correspondence of the input/output problem to the assembly of a structure with one less link.

Examples. Corollary 1 implies that a four-bar linkage has a coupler curve of degree less than or equal to 6 with bidegree no greater than 3 . It is well-known that a general RRRR four-bar coupler curve is in fact a tri-circular sextic, which means it attains both limits. All other four-bars, having one or more prismatic joints, fall inside the limits. For example, a slider-crank (RRRP) four-bar has a quartic coupler curve with bidegree 3 (Hunt 1978 p. 213-214), and a PRRP elliptic trammel (Cardan mechanism) has degree 2, bidegree 2. Theorem 1 implies that a tracing curve of a six-bar linkage has at most degree 20 and bidegree 10. As shown in (Primrose et al. 1967), the highest degree of any six-bar tracing curve is 18 (with bidegree 9 ), so the theorem is not sharp for $k \geq 3$.

\subsection{An eight-bar linkage}

Theorem 1 takes nothing into consideration beyond the number of links, so the bounds it predicts are not sharp. However, when applied to a particular linkage, the methodology often gives sharp results. To see this, consider the eight-bar linkage with tracing point $P$ shown in Figure 4(a). We wish to determine the degree and circularity of the tracing curve. The degree is equal to the number of assemblies of the structure shown in Fig. 4(b) and the bidegree is onehalf the number of assemblies of the structure in Fig. 4(c), because these will be the number of intersections of the coupler curve with a line and a circle, resp. By finding the number of assemblies of these structures, one also finds the degrees of the input/output relations for various tenbar linkages, in the manner discussed in $\$ 5.1$. Also, one finds the bidegree of other eight-bar linkages obtained from Fig. 4(d) by removing a binary link and declaring one of the adjacent links to be the ground link. For example, if link $B D$ is removed and link $D F G$ is held stationary, point $B$ will trace a coupler curve whose bidegree is the same as that of point $P$ in Fig. 4(a).

Numbering the links as shown in Fig. 4(d) and treating triangle $C P E$ as the fixed link, one may write the kinematic equations as 4 unit vector equations

$$
\theta_{1} \bar{\theta}_{1}=1, \quad \theta_{2} \bar{\theta}_{2}=1, \quad \theta_{3} \bar{\theta}_{3}=1, \quad \theta_{4} \bar{\theta}_{4}=1
$$

and 4 length equations

$$
\begin{aligned}
L_{1}^{2}= & \left(c+\theta_{1} a+\theta_{2} k-e-\theta_{3} g-\theta_{4} f\right) \\
& \cdot\left(\bar{c}+\bar{\theta}_{1} \bar{a}+\bar{\theta}_{2} \bar{k}-\bar{e}-\bar{\theta}_{3} \bar{g}-\bar{\theta}_{4} \bar{f}\right), \\
L_{2}^{2}=\left(c+\theta_{1} b-e-\theta_{3} g-\theta_{4} d\right)\left(\bar{c}+\bar{\theta}_{1} \bar{b}-\bar{e}-\bar{\theta}_{3} \bar{g}-\bar{\theta}_{4} \bar{d}\right), & \\
L_{3}^{2}=\left(c+\theta_{1} a+\theta_{2}(q+\ell)-e-\theta_{3} h\right) & \cdot\left(\bar{c}+\bar{\theta}_{1} \bar{a}+\bar{\theta}_{2}(\bar{q}+\bar{\ell})-\bar{e}-\bar{\theta}_{3} \bar{h}\right), \\
L_{4}^{2}= & \left(c+\theta_{1} a+\theta_{2} q\right)\left(\bar{c}+\bar{\theta}_{1} \bar{a}+\bar{\theta}_{2} \bar{q}\right) .
\end{aligned}
$$

This system of polynomials has the following productdecomposition form:

$$
\begin{aligned}
& f_{1} \in\left\langle\theta_{1}, 1\right\rangle\left\langle\bar{\theta}_{1}, 1\right\rangle \\
& f_{2} \in\left\langle\theta_{2}, 1\right\rangle\left\langle\bar{\theta}_{2}, 1\right\rangle \\
& f_{3} \in\left\langle\theta_{3}, 1\right\rangle\left\langle\bar{\theta}_{3}, 1\right\rangle \\
& f_{4} \in\left\langle\theta_{4}, 1\right\rangle\left\langle\bar{\theta}_{4}, 1\right\rangle \\
& f_{5} \in\left\langle\theta_{1}, \theta_{2}, \theta_{3}, \theta_{4}, 1\right\rangle\left\langle\bar{\theta}_{1}, \bar{\theta}_{2}, \bar{\theta}_{3}, \bar{\theta}_{4}, 1\right\rangle \\
& f_{6} \in\left\langle\theta_{1}, \theta_{3}, \theta_{4}, 1\right\rangle\left\langle\bar{\theta}_{1}, \bar{\theta}_{3}, \bar{\theta}_{4}, 1\right\rangle \\
& f_{7} \in\left\langle\theta_{1}, \theta_{2}, \theta_{3}, 1\right\rangle\left\langle\bar{\theta}_{1}, \bar{\theta}_{2}, \bar{\theta}_{3}, 1\right\rangle \\
& f_{8} \in\left\langle\theta_{1}, \theta_{2}, 1\right\rangle\left\langle\bar{\theta}_{1}, \bar{\theta}_{2}, 1\right\rangle
\end{aligned}
$$


Of the $2^{8}=256$ possible subsystems, there are $\left(\begin{array}{l}8 \\ 4\end{array}\right)=70$ ways of picking four factors for each of the two isotropic subsets. This 2-homogeneous Bezout number for the system is consistent with Theorem 1. However, some of these subsystems have no solutions. For example, the first factors from $f_{1}, f_{2}, f_{3}$ and $f_{7}$ cannot be chosen simultaneously as they give four general linear factors in only three unknowns. Altogether, there are 18 such ill-posed subsystems, leaving a maximum of 52 finite roots for the system. Consequently, the tracing curve for point $P$ in Fig. 4(a) has bidegree $b<26$ and degree $d<52$. When considering the linkage of Fig. 4(b), $f_{8}$ is replaced by $f_{8}^{\prime} \in\left\langle\theta_{1}, \theta_{2}, \bar{\theta}_{1}, \bar{\theta}_{2}, 1\right\rangle$, which also leads to $d<52$.

The inversion of the linkage to treat triangle $C P E$ as the fixed link was necessary to make the productdecomposition bound tight. If the same approach is attempted for the equations that derive naturally from the original non-inverted presentation, a bound of 54 is obtained instead of 52. In contrast, the BKK bound yields 52 in both cases. Because the BKK bound is completely automated, it may be the preferred methodology, especially for more complicated linkages. However, since a proof by hand is, in this case, more illuminating than a "black box" computation, the demonstration using product-decomposition has been presented.

To test if these bounds are tight, the system was solved with random link dimensions using 2-homogeneous polynomial continuation. This confirmed that both of the problems in question had 52 nonsingular finite roots. The analyst who is not interested in purist notions of mathematical proof could accept the numerical result as establishing the bound by itself, without the preceeding analytical treatment.

\section{SYNTHESIS OF LINKAGES}

Synthesis problems concern finding linkages whose motion will satisfy certain prespecified conditions, usually categorized as either function generation, curve generation, or body guidance problems. Since one may specify a variety of positional and/or rotational displacements of the links during the motion as well as giving various dimensions of the linkage, a complete catalog of synthesis problems would be too voluminous to be of much use. It is preferable to develop a unified approach to formulating and solving such problems. A formulation in isotropic coordinates, with the use of either product-decomposition or BKK theory to bound the number of roots, and the application of polynomial continuation to numerically solve for those roots, appears to be a promising approach. This contention is supported by the following two examples of synthesizing four-bar coupler curves.

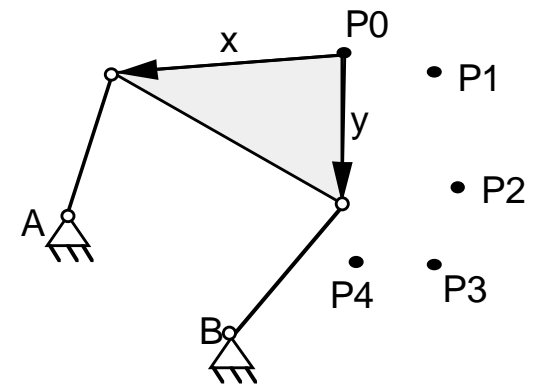

Figure 6. Five-point coupler-curve synthesis with prescribed pivots.

In the isotropic formulation of $\xi 2$, the link dimensions appear in isotropic coordinates as well as the motion variables. These dimensions become variables in a synthesis problem, so isotropic coordinates will once again be an aid in eliminating certain kinds of roots at infinity that relate to circularity.

\subsection{A five-point problem}

Consider the problem from Morgan and Wampler (1990) of constructing a four-bar linkage whose coupler point passes through 5 given points $P_{0}, \ldots, P_{4}$ and whose fixed pivots $A, B$ are given (Fig. 6). Let $x$ and $y$ be complex vectors from $P_{0}$ to the moving pivots in the initial position of the linkage, and for positions $j=1,2,3,4$, let $\theta_{j}, \bar{\theta}_{j}$ be isotropic coordinates for the rotation of the coupler link relative to its initial position. For each of $j=1,2,3,4$, there is one unit vector equation and one equation for each binary link, expressing the fact that its length is constant:

$$
\begin{aligned}
\theta_{j} \bar{\theta}_{j} & =1, \\
\left(p_{j}+\theta_{j} x-a\right)\left(\bar{p}_{j}+\bar{\theta}_{j} \bar{x}-\bar{a}\right) & =\left(p_{0}+x-a\right)\left(\bar{p}_{0}+\bar{x}-\bar{a}\right), \\
\left(p_{j}+\theta_{j} y-b\right)\left(\bar{p}_{j}+\bar{\theta}_{j} \bar{y}-\bar{b}\right) & =\left(p_{0}+y-b\right)\left(\bar{p}_{0}+\bar{y}-\bar{b}\right),
\end{aligned}
$$

where $\left(p_{j}, \bar{p}_{j}\right)$ are the isotropic coordinates for $P_{j}$, and similarly for $A$ and $B$. The monomials appearing in these equations are

$$
\begin{gathered}
\left\langle\theta_{j} \bar{\theta}_{j}, 1\right\rangle, \\
\left\langle\theta_{j} x, \bar{\theta}_{j} \bar{x}, x, \bar{x}, 1\right\rangle, \\
\left\langle\theta_{j} y, \bar{\theta}_{j} \bar{y}, y, \bar{y}, 1\right\rangle .
\end{gathered}
$$

Since $(18,19)$ are linear in $\theta_{j}, \bar{\theta}_{j}$, such equations can be solved by Cramer's rule. When the results are substituted into (17), one obtains quartics

$$
f_{j} \in\langle x \bar{y}, \bar{x} \bar{y}, \bar{x} y, \bar{x}, \bar{y}\rangle\langle x y, x \bar{y}, \bar{x} y, x, y\rangle, \quad j=1,2,3,4 .
$$


(If we treat $x, \bar{x}, y, \bar{y}$ as constants and $p_{j}, \bar{p}_{j}$ as variables, each of these is a four-bar coupler curve equation of degree 6 , bidegree 3. )

There are several ways to treat this problem. Morgan and Wampler (1990), working in Cartesian coordinates, derived a system of 4 quartics equivalent to Eq.(20). Although the 2-homogeneous Bezout number was 96, upon solving the problem numerically with polynomial continuation for general $P_{j}, A$, and $B$, they observed that 60 roots always fall on a pair of degenerate hyperplanes. This leaves at most 36 finite, nonsingular roots. By the method of parameter continuation (Morgan and Sommese 1989), they were able to construct a 36-path homotopy for solving subsequent instances of the problem.

While the numerical approach provides compelling evidence that the Bezout number for the problem is 36 , it does not provide proof. An alternative is to compute the BKK bound of the system, which for any system of the form Eqs.(17-19) in 12 variables, or Eqs.(20) in 4 variables, is 36. Accordingly, the approaches discussed in (Verschelde et al. 1994, Huber and Sturmfels 1995) can be used to solve the problem via a 36-path homotopy. It is important to note that the BKK bound for the 4 quartics in Cartesian coordinates is 80 , which shows the value of an isotropic formulation. This is because in isotropic coordinates, the degenerate sets are $(x, y)=(0,0)$ and $(\bar{x}, \bar{y})=(0,0)$. Since the BKK formula counts roots in $(C-\{0\})^{n}$, these sets are ignored.

A third alternative is to analyze the problem using the product decomposition. This approach is not as automated as the previous two, but it does provide a methodology that can be carried out by hand to prove the bound of 36 . The system represented by Eq.(20) breaks naturally into $2^{4}$ subsystems. These come in 5 types, according to whether the first factor is chosen $0,1,2,3$, or 4 times. Because of the symmetry in the structure of the two factors, type 0 and type 4 subsystems will have the same number of roots, and the same holds for type 1 and type 3 subsystems. Thus, only 3 subsystems need to be considered to find a Bezout number for the full system. Since four-bars with $\bar{x}=0$ or $\bar{y}=0$ are not of interest, we can divide the equations by $\bar{x}^{2} \bar{y}^{2}$ to obtain polynomials that belong to

$$
\langle s, 1, t, u, v\rangle\langle s t, s, t, s u, t v\rangle
$$

where

$$
s=x / \bar{x}, \quad t=y / \bar{y}, \quad u=1 / \bar{y}, \quad v=1 / \bar{x} .
$$

For a given value of $(s, t, u, v)$, there is one value of $(x, y, \bar{x}, \bar{y})$. The (unique) type 4 system, which consists of 4 linear polynomials of the form $\langle s, 1, t, u, v\rangle$, has at most 1 solution. A type 3 subsystem consists of 3 linear and one quadratic polynomial, so it has at most 2 nonsingular solutions. A type 2 system has at most 4 solutions, but one of these is of the form $(s, t)=(0,0)$, so we do not count it. Hence, the total number of nonsingular, nonzero roots is at most

$$
\left(\begin{array}{l}
4 \\
4
\end{array}\right) 1+\left(\begin{array}{l}
4 \\
3
\end{array}\right) 2+\left(\begin{array}{l}
4 \\
2
\end{array}\right) 3+\left(\begin{array}{l}
4 \\
1
\end{array}\right) 2+\left(\begin{array}{l}
4 \\
0
\end{array}\right) 1=36
$$

This establishes the desired result. A 36-path homotopy could be created using this approach.

\subsection{The nine-point problem}

Since a four-bar coupler curve has nine independent parameters, one can specify up to nine general points on the curve. This problem can be formulated exactly as in the five-point problem above, except $a, \bar{a}, b, \bar{b}$ become variables and there are nine precision points $P_{0}, \ldots, P_{8}$. This problem was formulated by Alt (1923), with the first partial solutions found by Roth and Freudenstein (1963). Their "bootstrap" technique was a precursor to the methodology now known as "polynomial continuation," which was used in conjuction with an isotropic formulation by Wampler et al. (1992) to find complete solutions. The computation of the BKK bound for this system is reported in Verschelde et al (1995) and an improved treatment based on the product decomposition appears in Morgan et al (1995).

\section{CONCLUSIONS}

The isotropic formulation of kinematic equations facilitates the solution of problems concerning the analysis and synthesis of planar mechanisms. It has been shown that isotropic coordinates are intimately related to issues of circularity and in fact, the concept of circularity can be supplanted by isotropic bidegree, which simplifies many derivations. An isotropic formulation also submits readily to treatments utilizing several modern root-counting methods, namely multi-homogeneous Bezout numbers, the BKK bound, and the product-decomposition theorem. Using this synergistic combination of formulation and analytical tools, upper bounds on the degree and bidegree of the curves traced by planar linkages have been established. The close relation between these bounds, the number of assemblies of structures, and the input/output degree of linkages has been elucidated. The applicability of the methods has been demonstrated in the analysis of an eight-bar linkage having a coupler curve of degree 52 and in the synthesis of fourbar coupler curves. While this paper has concentrated on analytical aspects of the problem, all the methods lead di- 
rectly to numerical implementations based on polynomial continuation.

Many questions have been left open. In particular, the analysis of tracing curves has been limited to the study of their degree and circularity. Questions of genus and of the number of double points, cusps, and real circuits, while of interest, are not addressed. The analysis of RP- and PP-type coupler mechanisms also awaits attention. As for synthesis problems, wide regions of territory remain unexplored. Here there will be challenge not just in solving particular combinations of precision data, but also in identifying which of these might be effective tools for practical linkage design.

\section{ACKNOWLEDGMENT}

The author is grateful to J. Verschelde, Katholieke Universiteit Leuven, for providing a copy of the PHC code, with which all computations of BKK numbers in this paper were accomplished.

\section{REFERENCES}

Alt, H., 1923, "Über die erzeugung gegebener ebener kurven mit hilfe des gelenkvierseits," $Z$. Angew. Math. und Mech., Vol. 3, pp. 13-19.

Bernshtein, D.N., 1975, The number of roots of a system of equations, Funktsional. Anal. i Prilozhen., Vol. 9, No. 3, pp. 1-4 (translated).

Bricard, R., 1927, Leçons de Cinématique, Tome II, pp. $301-311$.

Darboux, G., 1879, "De l'emploi des fonctions elliptiques dans la théorie du quadrilatère plan," Bull. des Sciences, math. et astronom. 2e serie T. III, pp. 109-128.

Groenman, J.T., 1950, "Behandeling van de koppelkromme met behulp van isotrope coördinaten," Ph.D. Thesis, Technische Hogeschool te Delft.

Haarbleicher, A., 1933, "Application des coordonnées isotropes a l'étude de la courbe des trois barres," J. de l'Ecole Polytechnique, II serie, Vol. 31, pp. 13-40.

Huber, B., and Sturmfels, B., 1995, "A polyhedral method for solving sparse polynomial systems," Math. Comp., to appear.

Hunt, K.H., 1978, Kinematic geometry of mechanisms, Oxford Univ. Press, Oxford.

Morgan, A.P., and Sommese, A..J., 1987, "A homotopy for solving general polynomial systems that respects $m^{-}$ homogeneous structures," Applied Math. and Comp., Vol. 24, pp. 101-113.

Morgan, A.P., and Sommese, A.J., 1989, "Coefficientparameter polynomial continuation," Applied Math. and Comp., Vol. 29, pp. 123-160.
Morgan, A.P., Sommese, A.J., and Wampler, C.W., 1995, "A product-decomposition bound for Bezout numbers," SIAM J. Numer. Anal., Vol. 32, pp. 1308-1325.

Morgan, A.P., and Wampler, C.W., 1990, "Solving a planar four-bar design problem using continuation," ASME J. Mechanical Design, Vol. 112, pp. 544-550.

Morley, F.V., 1921, "An analytical treatment of the 3 bar curve," Proc. London Math. Soc., Vol. 21, pp. 140-160.

Primrose, E.J.F., and Freudenstein, F., 1963, "Geared five-bar motion: Part 2-Arbitrary commensurate gear ratio," Trans. ASME Series E (J. Applied Mechanics), Vol. 30E, pp. 170-175.

Primrose, E.J.F., Freudenstein, F., and Roth, B., 1967, "Six-bar motion (Parts I-III)," Archive for Rational Mechanics and Analysis, Vol. 24, pp. 22-77.

Roberts, S., 1875, "On three-bar motion in plane space," Proc. London Math. Soc., Vol. VII, pp. 14-23.

Roth, B., and Freudenstein, F., 1963, "Synthesis of path-generating mechanisms by numerical methods," Trans. ASME Series B (J. Eng. Ind.), Vol. 85B, No. 3, pp. $298-306$.

Shafarevich, I.R., 1977, Basic Algebraic Geometry, Springer, New York.

Smith, J.A., 1975, "Kinematic analysis of mechanisms by the complex conjugate exponential method," Trans. ASME Series B (J. Eng. Ind.), Vol. 97B, pp. 795-800.

Verschelde, J., Gatermann, K., and Cools, R., 1995, "Mixed volume computation by dynamic lifting applied to polynomial system solving," Discrete Comput. Geom, to appear.

Verschelde, J., Verlinden, P., and Cools, R., 1994, "Homotopies exploiting Newton polytopes for solving sparse polynomial systems," SIAM J. Numer. Anal., Vol. 31, No. 3, pp. 915-930.

Wampler, C.W., Morgan, A.P., and Sommese, A.J., 1992, "Complete solution of the nine-point path synthesis problem for four-bar linkages," J. Mechanical Design, Vol. 114 , p. $153-159$. 